ИЗВЕСТИЯ АКАДЕМИИ НАУК ЭСТОНСКОИ ССР, ТОМ 26 ФИЗИКА * МАТЕМАТИКА. 1977, № 4

\title{
ЧАСТОТА БУКВ В ПЕЧАТНОМ ТЕКСТЕ НА ЭСТОНСКОМ ЯЗЫКЕ
}

\section{Введение}

При синтезе речи по печатному тексту буквы преобразуются в акустические волны речевых сигналов.

В синтезе слитной речи участвуют сочетания различных фонем, их составляющие характеризуются своими определенными значениями параметров (частотой, амплитудой и шириной формант, частотой основного тона, спектром и амплитудой шума и т. д.). При произнесении гласных и сонорных согласных различаются три этапа - появление, квазистационарная часть и исчезновение. Слитность произношения вызывает взаимное влияние смежных фонем друг на друга. Поэтому при разработке законов управления параметрами синтезаторов речевых сигналов необходимо располагать данными о существующих сочетаниях фонем, выраженных в печатном тексте буквами.

Статистическим анализом текстов на эстонском языке занимались несколько авторов. В [1,2] были определены частота появления в словах отдельных букв и комбинаций слогов из двух и трех букв. Однако в разговоре отдельные слова сливаются и поэтому воспринимаются слухом как последовательность звуковых единств, т. е. синтагмами, паузы между которыми на письме выделяются, как правило, знаками препинания. Поэтому при вводе печатного текста для обработки в ЭВМ знаки препинания (запятые, точки, двоеточия, точки с запятой, вопросительные и восклицательные знаки, кавычки, скобки, тильды и тире, а также обрывы слитности при чтении) должны кодироваться как паузы.

Материалом для анализа служили произвольно выбранные отрезки текстов из книг, журналов и газет в соотношении

$$
\begin{array}{ll}
\text { научно-техническая литература } & 47,1 \% \\
\text { художественная литература } & 22,1 \% \\
\text { общественно-публицистическая } & 30,8 \%
\end{array}
$$

Изучено было 102307 букв. Исследование по синтагмам давало больше комбинаций букв по сравнению с исследованием по словам, поскольку в речевом потоке слова сливаются и произносятся с паузами только после нескольких слов.

В алфавите эстонского языка 23 буквы. В написаниях имен и в заимствованиях встречаются, кроме того, буквы $c, f, q, \check{s}, z, \check{z}, w, x$ и $y$. Из них мы учитывали только $f$, как наиболее употребительную. 
Таблица 1

\begin{tabular}{|c|c|c|c|c|}
\hline Буква & Частота & $\begin{array}{c}\text { Относитель- } \\
\text { ная частота, } \\
\% \\
\end{array}$ & & $\begin{array}{l}\text { ть- } \\
\text { щы, }\end{array}$ \\
\hline$a$ & 12148 & 11,87 & 11,61 & 12,13 \\
\hline$e$ & 12086 & 11,81 & 11,55 & 12,07 \\
\hline$i$ & 10278 & 10,05 & 9,81 & 10,29 \\
\hline$s$ & 9389 & 9,18 & 8,95 & 9,42 \\
\hline$t$ & 8012 & 7,83 & 7,62 & 8,05 \\
\hline$l$ & 5966 & 5,83 & 5,64 & 6,02 \\
\hline$u$ & 5855 & 5,72 & 5,54 & 5,91 \\
\hline$m$ & 4599 & 4,50 & 4,67 & 4,34 \\
\hline$k$ & 4518 & 4,42 & 4,26 & 4,59 \\
\hline$n$ & 4513 & 4,41 & 4,25 & 4,58 \\
\hline$d$ & 3868 & 3,78 & 3,63 & 3,94 \\
\hline$o$ & 3852 & 3,76 & 3,61 & 3,92 \\
\hline$r$ & 3414 & 3,34 & 3,20 & 3,49 \\
\hline$v$ & 2434 & 2,38 & 2,26 & 2,51 \\
\hline$g$ & 1863 & 1,82 & 1,72 & 1,93 \\
\hline$\vec{j}$ & 1738 & 1,70 & 1,60 & 1,81 \\
\hline$h$ & 1623 & 1,59 & 1,49 & 1,69 \\
\hline$p$ & 1611 & 1,57 & 1,47 & 1,67 \\
\hline$\tilde{o}$ & 1244 & 1,22 & 1,13 & 1,31 \\
\hline$\ddot{a}$ & 1240 & 1,21 & 1,12 & 1,30 \\
\hline$b$ & 818 & 0,80 & 0,73 & 0,88 \\
\hline$\ddot{u}$ & 781 & 0,76 & 0,69 & 0,83 \\
\hline$\ddot{o}$ & 374 & 0,37 & 0,32 & 0,42 \\
\hline$f$ & 83 & 0,08 & 0,06 & 0,11 \\
\hline cero & 102307 & 100,00 & & \\
\hline
\end{tabular}

\section{Результаты исследования}

Данные изучения частотности отдельных букв в печатном тексте приведены в табл. 1. Как видно, относительная частота встречаемости всех девяти гласных составляет $46,8 \%$, причем наиболее употребительны $a, e$ и $i-33,7 \%$ всех букв и $71,4 \%$ всех гласных. Среди согласных наиболее распространена буква $s$. Взрывная $t$ встречается почти в пять раз чаще, чем $p$, и почти в два раза чаще, чем $k$. Общий процент повторяемости $t, p$ и $k$ в два раза выше такового $b, d$ и $g$. Гласная $i$ встречается в шесть раз чаще полугласного $j$, а если считать, что они описывают одну и ту же фонему, то вероятность их появления вместе взятых приближается к вероятности появления $a$ и $e$.

В табл. 2 представлены данные о частотности диад, т. е. двухбуквенных сочетаний. По горизонтали выписаны первые и по вертикали вторые буквы диад. Всего выявлено 94010 сочетаний. В отдельных словах эстонского языка используются 25 комбинаций гласных: $a e, a i, a о$, $a u, e a, e i, e o, i o, i u, o a, o e, o i, o u, u i, \tilde{o} a, \tilde{o} e, \tilde{o} i, \tilde{o} o, \tilde{o} u, \ddot{a} e, a ̈ i, a ̈ o, a ̈ u$, $\ddot{o} е$ и $\ddot{o} i$. В слитной же речи их количество увеличивается до 60, т. е. по сравнению с отдельными словами становится больше, чем в два раза. Теоретически из девяти гласных можно образовать 72 варианта. Значит, в синтагмах встречается $83,2 \%$ возможных сочетаний, причем гласные $a, e, i$ и $u$ имеют все комбинации. Из 24 букв (23 эстонских плюс $f$ ) теоретически можно сформировать 552 двухбуквенные комбинации. В синтапмах обнаружено 504 разные диады, т. е. 90,9\% теоретически возможных. Из них чаще десяти раз появилось 397 диад и чаще пяти раз 442 диады. Последние составляют $78,3 \%$ их общего количества.

Исследованы частоты встречаемости триад, т. е. комбинации из трех 
๙

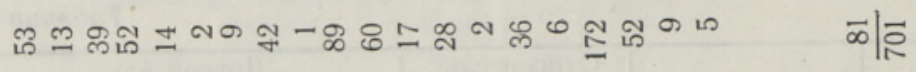

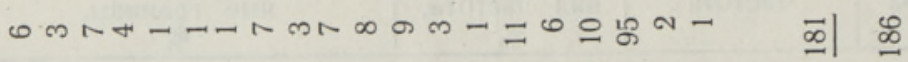
サナமッ -

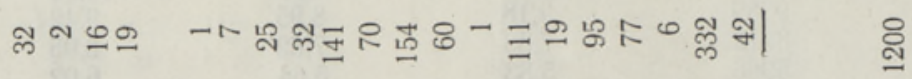
추ำ

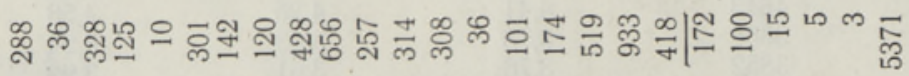

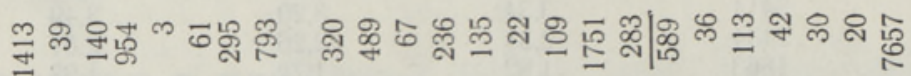
ํㅗㄱ ₹ 궁 ลิ 茫 ஜ

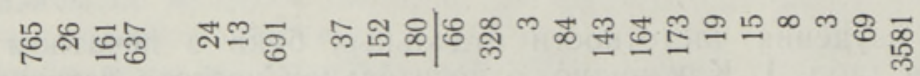

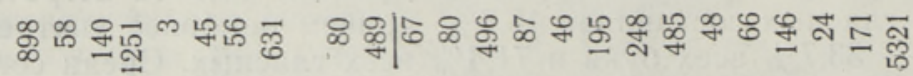

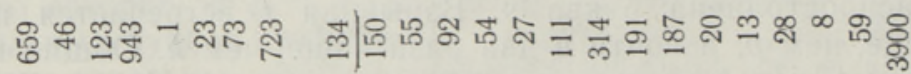
尔的娄

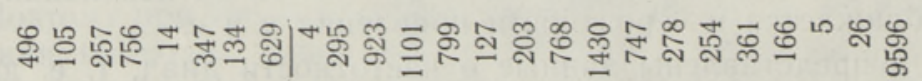
ల్లి ลักิ

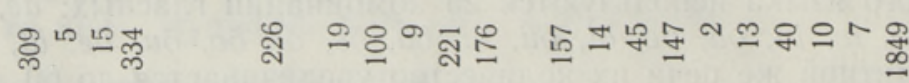

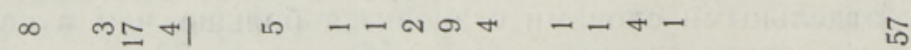

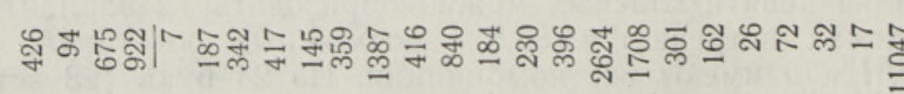
赵

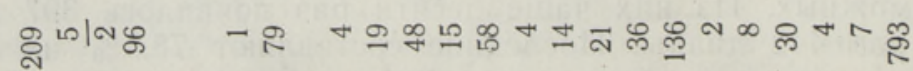

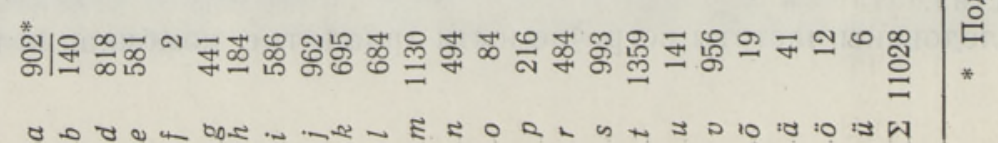




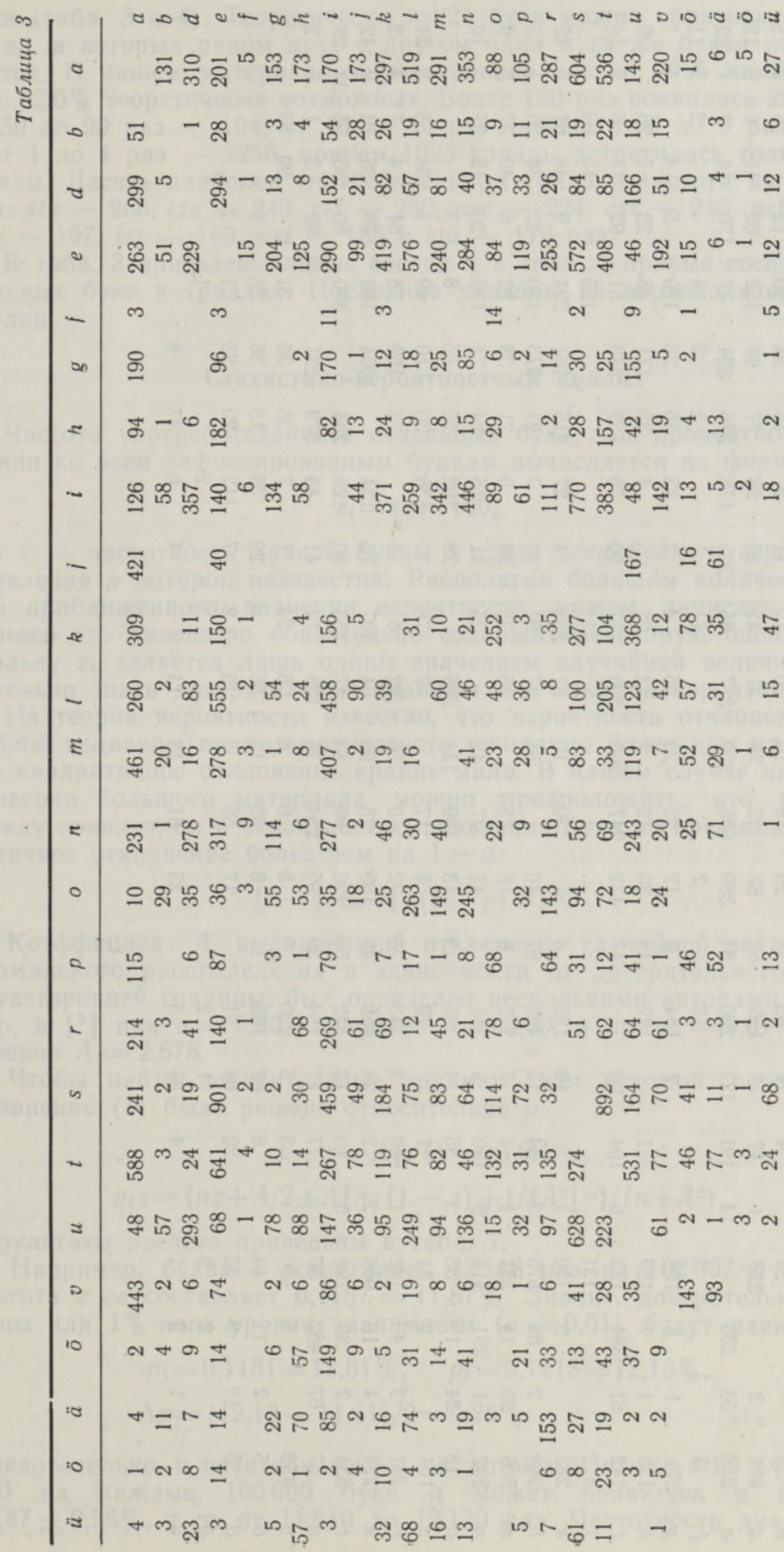




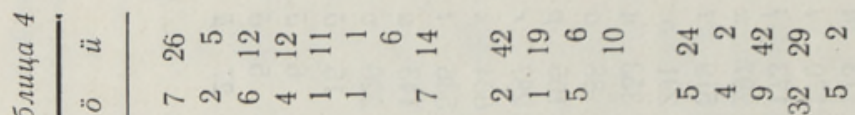

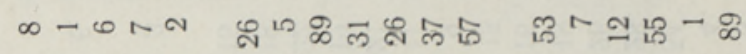
으부는 웅요 으ำํำ 으으ำ

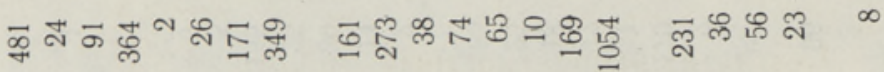

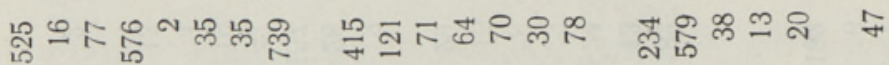

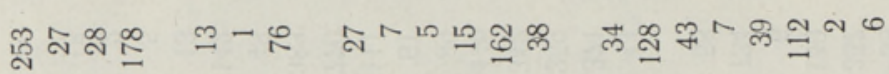

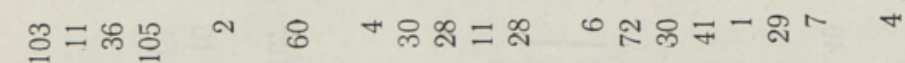
எㅇำ

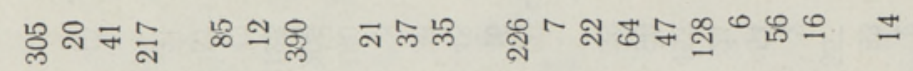

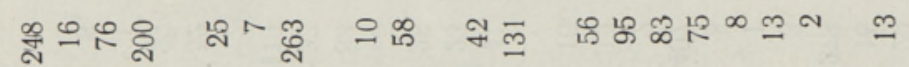

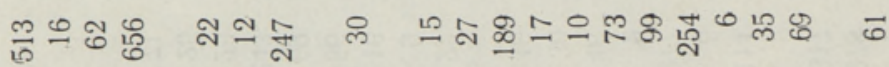

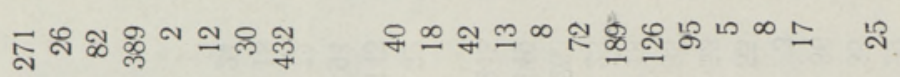

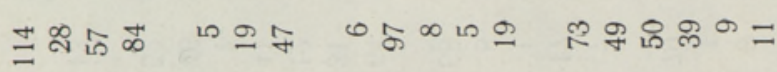

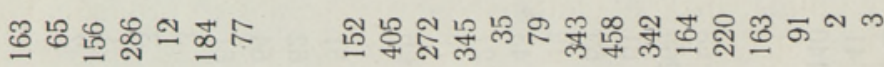
ஐサーล ๓ัニ $+- \pm \quad \infty-\infty-\infty m-\infty$ 늠ํํ ๓

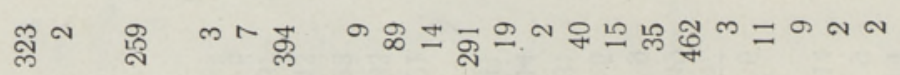

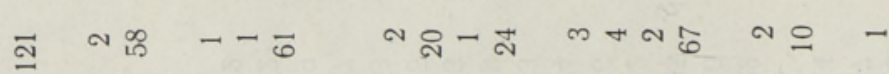

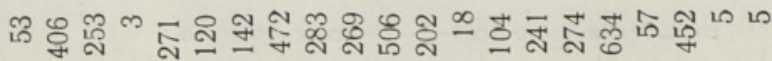

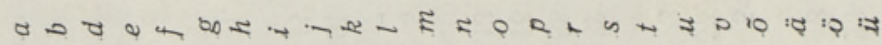


букв (табл. 3 и 4). Теоретически из 24 букв можно образовать 12696 триад, в которых рядом друг с другом одна и та же буква не повторяется. В нашем материале зафиксировано только 4065 комбинаций, т. е. $32,0 \%$ теоретически возможных. Более 100 раз появились 29 триад, от 50 до 99 раз - 104, от 10 до 49 раз - 936, от 5 до 9 раз - 740 и от 1 до 4 раз - 2256, пришем 1023 триады встретились только единожды. Десять наиболее употребительных триад и частота их появления: ste -266 , ise -240 , rel -230 , use -224 , est -216 , ast -210 , mis -197 , ist -189 , ust -185 и sta -179 раз.

В табл. 3 приведены левые соседи и в табл. 4 правые соседи центральных букв в триадах. Последние указаны на вертикальной линии таблиц.

\section{Статистико-вероятностный анализ}

Частота употребительности отдельных букв $z_{i}$ в процентном выражении ко всем зафиксированным буквам вычисляется по формуле

$$
z_{i}=x_{i} / n \cdot 100,
$$

где $x$ - частотность данной буквы $i$ среди всех букв $n$, вероятность появления $p$ которой неизвестна. Располагая большим количеством $n$, для приближенного значения вероятности можем записать $p_{i} \approx z_{i}$. Однако это равенство обязательно содержит некоторую ошибку. Поскольку $z_{i}$ является лишь одним значением случайной величины, желательно знать доверительные границы для искомой вероятности.

Из теории вероятности известно, что вероятность отклонения случайной величины от математичеокого ожидания более чем на утроенное квадратичное отклонение крайне мала. В нашем случае на основе проверки большого материала можно предположить, что разность между появлением и вероятностью появления букв не превышает квадратичное отклонение более чем на $1-\alpha$ :

$$
|z-p| \leqslant A[p(1-p) / n]^{1 / 2} \text {. }
$$

Коэффициент $A$, выражающий отклонение случайной величины от нормального распределения в зависимости от доверительного уровня двухсторонней границы, был определен несколькими авторами. Например, в $\left[{ }^{3}\right]$ при $\alpha=0,002$ получено $A=3,09$, а при $\alpha=0,01$ соответственно $A=2,578$.

Чтобы найти доверительные границы для искомой вероятности, уравнение (1) было решено относительно $p$ :

$$
\begin{gathered}
z-p=A[p(1-p) / n]^{1 / 2} \\
p_{1,2}=\left\{n z+A / 2 \pm A\left[n z(1-z)+1 / 4 A^{2}\right]^{1 / 2}\right\} /\left(n+A^{2}\right) .
\end{gathered}
$$

Результаты расчета приведены в табл. 1.

Например, буква а встретилась 12148 раз на 102307 букв, т. е. частота $z$ ее составляет $0,1187=11,87 \%$. Значит, доверительные границы для $1 \%$-ного уровня значимости $(\alpha=0,01)$ будут равны

$$
\begin{aligned}
& p_{1}=0,1161=11,61 \%, \quad p_{2}=0,1213=12,13 \%, \\
& \Delta p=(12,13-11,61) / 2=0,26 \% .
\end{aligned}
$$

Следовательно, в печатном тексте на эстонском языке в 99 случаях из 100 на каждые 100000 букв $a$ может появиться в пределах $11,87 \pm 0,26 \%$, т. е. от 11610 до 12130 раз. Частотность диад значи- 
тельно меньше. Из всех букв 94010 диад наиболее употребительна диада se - 2624 раза. Доверительные границы для $1 \%$-ного уровня значимости $(\alpha=0,01)$ равны $p_{1}=2,93 \%$ и $p_{2}=2,66 \%$, т. е. диада se может встретиться в тексте из 100000 букв от 2496 до 2757 раз.

\section{Заключение}

Проведен статистический анализ частотности появления отдельных букв, двух- и трехбуквенных сотетаний в произвольно выбранных фрагментах печатного текста на эстонском языке. Установлено, что наиболее часто встречаются буква $a$, диада se и триада ste. Слово состоит в среднем из 7 букв и синтагма из 35 букв. Статистика показала, что в слитной речи появляются сочетания букв, отсутствующие в отдельных словах. Результаты исследования будут использованы при разработке законов управления параметрами синтезатора речевых сигналов по печатному тексту.

\section{Л И Т Е Р А Т У Р А}

1. Ka a sik, D., L a ug a ste, E., Keel ja kirjandus, 12, 600 (1969).

2. K a a s i k, U., L a u g a s te, E., $\AA$ är e ma a, K., Keel ja kirjandus, 18, 21 (1975).

3. Fisher, R. A., Statistische Methoden für die Wissenschaft, Edinburgh, 1956.
Ннститут кибернетики
Академии наук Эстонской ССР
Поступила в редакцию 10/XI 1976

\section{E. KONNAP}

\section{TÄHTEDE ESINEMISSAGEDUS EESTIKEELSES TEKSTIS}

Tuuakse andmeid tähtede ning kahe- ja kolmetäheliste kombinatsioonide esinemissageduse kohta eestikeelsetes tekstides. Uurimise tulemusi kasutatakse trükiteksti-kõnesüntesaatori juhtimissüsteemide väljatöötamisel.

\section{E. KONNAP}

\section{FREQUENCY OF OCCURRENCE OF LETTERS IN WRITTEN ESTONIAN}

Statistical information was obtained about written Estonian. The frequency of occurrence of letters, dyads and triads is presented. The investigation results will be used for working out rules for control of the synthesizers when synthesizing speech from a written text. 